\title{
Bit Error Rate Analysis for V-BLAST/STBC using Interference Cancellation Techniques
}

\author{
Chirawat Kotchasarn \\ Department of Electronics and Telecommunications Engineering, \\ Rajamangala University of Technology Thanyaburi, Pathumthnani, Thailand, 12110 \\ Tel: (662) 549-4620 Fax: (662) 549-4622 \\ E-mail: chirawat.k@en.rmutt.ac.th
}

\begin{abstract}
Multiple Input Multiple Output (MIMO) systems have been extensively studied in context of wireless communication systems, which promising the both increased capacity and link level reliability. In this paper, we proposed the interference cancellation techniques for $\mathrm{V}$ BLAST/STBC architecture, which integrates Alamouti space-time block code (STBC) layer together with vertical Bell-laboratories (V-BLAST) layer. In this work, we employ four transmit and three receive antennas over frequency flat Rayleigh fading channel. This paper presents signal detectors for $\mathrm{V}$-BLAST/STBC with minimum mean square error (MMSE), zero forcing (ZF), parallel interference cancellation (PIC) and successive interference cancellation-zero forcing with binary phase shift keying (BPSK). Simulation results show the bit error rate performance degradation and the computational complexity of the joint optimal detection is better than the other detection technique.
\end{abstract}

Keywords- interference cancellation; Vertical Bell Laboratories Layered Space-Time; Space Time Block Code; zero forcing; minimum mean square error; successive interference cancellation; parallel interference cancellation

\section{INTRODUCTION}

In wireless communication systems, continuous communication with high quality of signal that contains voice, text, or image, is not guaranteed because channel condition can be not good owing to multipath fading channel [1]. Also, wireless communication systems face significant design challenges to achieve the desired performance and coverage range due to limitations on the transmit power level [2]. One method for overcome these problems is adopting analog repeaters. But the repeaters amplify both the signal and noise. The possible solution is used multiple antennas, which called multiple-input multiple-output (MIMO) [3]. This technique can effectively exploit the spatial diversity, time diversity and frequency diversity. Therefore, the coverage area and the system performance are significantly improved. Vertical Bell Labs Layered SpaceTime (V-BLAST) was proposed [4] to achieve the very high spectral efficiency promised by the multiple-antenna system [5]-[7].
In the original V-BLAST system [4], parallel data streams are simultaneously transmitted through multiple antennas in the same frequency band, which helps to achieve the high spectral efficiency with reasonable decoding complexity. The performance analysis of V-BLAST using ZF-SIC detector is in general considered difficult. The difficulty mainly comes from the nonlinear interference cancellation operation, which generates the so-called error propagation effect in practical systems and is hard to quantify. Preliminary research in [8]-[9] has reported the asymptotic analysis and numerical Monte Carlo simulation results. Although the numerical approach is useful in performance evaluation, the analytical approach provides deep insight and comprehensive understanding of the essential and key points of V-BLAST. Furthermore, analytical results are useful in developing optimal transmission schemes such as power allocation. [10] derives closed-form expressions for signals at each detection step and performs statistical analysis in a Rayleigh-fading channel based on the perfect interference cancellation assumption. [11] breaks this assumption and presents an analysis on the joint error rate and symbol error rate. However the closed-form expression of bit error rate (BER) of each substream is still unknown.

A V-BLAST/ STBC scheme which contains Alamouti space time block codes (STBC) with V-BLAST is consider in this paper. This scheme arises as a solution to jointly achieve spatial multiplexing and diversity gains simultaneously. With VBLAST/STBC scheme, it is possible to increase the data rate while keeping a satisfactory link quality in terms of the symbol error rate (SER). Due to the error propagation effect, the detection error of the first sub-stream can result in more errors than the subsequent substreams detection. Hence the first substream limits the overall performance of this structure. In this work a joint SIC-ZF detection algorithm is proposed to reduce the system complexity and minimize the performance degradation.

This paper is organized as follows. In Section II, the VBLAST/STBC architecture is presented. In section III, we proposed the interference cancellation techniques with linear receiver such as MMSE, ZF and non-linear receiver such as PIC, SIC-ZF. The system performance is also included in section III. The simulation results are described in section IV. Finally, section $\mathrm{V}$ provides the conclusion. 


\section{SYSTEM MODEL}

The architecture of V-BLAST/STBC with four transmitters and three receivers has been described in Fig.1

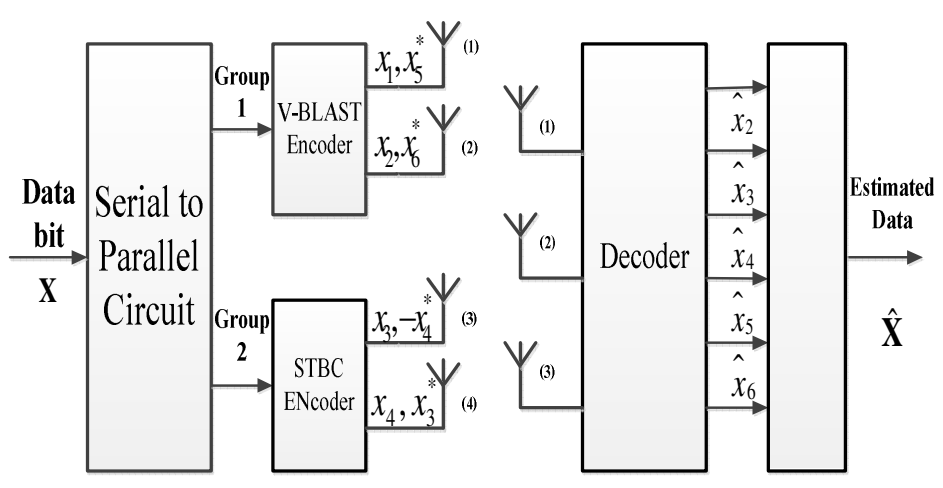

Figure 1. V-BLAST/STBC transceiver.

Let the data transmission bits denoted by $\mathbf{X}$, which is a serial data using serial to parallel circuit. All of the data streams are demultiplexed into two groups as known as V-BLAST and STBC. The data bits $x_{1}$ and $x_{5}^{*}$ are transmitted by the first antenna of V-BLAST at time $t$ and $t+T$. The data bit $x_{2}$ and $x_{6}^{*}$ are transmitted by the second antenna of V-BLAST at time $t$ and $t+T$, respectively. The data bit $x_{3}$ and $-x_{4}^{*}$ are transmitted by STBC at time $t$ and $t+T$. Finally, the data bits $x_{4}$ and $x_{3}^{*}$ are transmitted by STBC at time $t$ and $t+T$, respectively. Each signal is assumed to be binary phase shift keying (BPSK). The channel model is described as Rayleigh frequency flat fading channel and assumed be a constant across two consecutive symbol transmission periods. It is denoted by $h_{i j}$ between the transmit antenna $i$ and receive antenna $j$. Each entries of $h_{i j}$ are independent and identically distributed and circularly symmetric Gaussian random variables with zero-mean and unit variance. The receive signal form the transmit antenna $a$ to the receive antenna $b$ at time $t$ and $t+T$ is denoted by $y_{a b}$. Finally, $n_{i j}$ is denoted as the additive white Gaussian noise (AWGN) with zero mean and variance $\sigma^{2}$ between the $i$ transmit antenna and the $j$ receive antenna. Therefore, the received signals at the first receive antenna at time $t$ and $t+T$ are given by

and

$$
y_{11}=h_{11} x_{1}+h_{12} x_{2}+h_{13} x_{3}+h_{14} x_{4}+n_{11}
$$

$$
y_{12}=h_{11} x_{5}^{*}+h_{12} x_{6}^{*}+h_{13}\left(-x_{4}^{*}\right)+h_{14} x_{3}^{*}+n_{12} \text {. }
$$

In the same manner, the received signals at the second and third receive antennas at time $t$ and $t+T$ are described by

$$
\begin{array}{ll}
y_{21} & =h_{21} x_{1}+h_{22} x_{2}+h_{23} x_{3}+h_{24} x_{4}+n_{21}, \\
y_{22} & =h_{21} x_{5}^{*}+h_{22} x_{6}^{*}+h_{23}\left(-x_{4}^{*}\right)+h_{24} x_{3}^{*}+n_{22}, \\
y_{31} & =h_{31} x_{1}+h_{32} x_{2}+h_{33} x_{3}+h_{34} x_{4}+n_{31}, \\
\text { and } \quad & y_{32}=h_{31} x_{5}^{*}+h_{32} x_{6}^{*}+h_{33}\left(-x_{4}^{*}\right)+h_{34} x_{3}^{*}+n_{32} .
\end{array}
$$

These signals can be written in a matrix form as $\left[\begin{array}{ll}y_{11} & y_{12} \\ y_{21} & y_{22} \\ y_{31} & y_{32}\end{array}\right]=\left[\begin{array}{llll}h_{11} & h_{12} & h_{13} & h_{14} \\ h_{21} & h_{22} & h_{23} & h_{24} \\ h_{31} & h_{32} & h_{33} & h_{34}\end{array}\right]\left[\begin{array}{cc}x_{1} & x_{5}^{*} \\ x_{2} & x_{6}^{*} \\ x_{3} & -x_{4}^{*} \\ x_{4} & x_{3}^{*}\end{array}\right]+\left[\begin{array}{ll}n_{11} & n_{12} \\ n_{21} & n_{22} \\ n_{31} & n_{32}\end{array}\right] .(7)$
Let $\mathbf{y}=\left[\begin{array}{ll}y_{11} & y_{12} \\ y_{21} & y_{22} \\ y_{31} & y_{32}\end{array}\right]$ be a received signal matrix. The Rayleigh fading matrix is denoted by $\mathbf{h}=\left[\begin{array}{llll}h_{11} & h_{12} & h_{13} & h_{14} \\ h_{21} & h_{22} & h_{23} & h_{24} \\ h_{31} & h_{32} & h_{33} & h_{34}\end{array}\right]$ $\mathbf{x}=\left[\begin{array}{cc}x_{1} & x_{5}^{*} \\ x_{2} & x_{6}^{*} \\ x_{3} & -x_{4}^{*} \\ x_{4} & x_{3}^{*}\end{array}\right]$ is a transmit signal matrix and $\mathbf{n}=\left[\begin{array}{cc}n_{11} & n_{12} \\ n_{21} & n_{22} \\ n_{31} & n_{32}\end{array}\right]$ is an AWGN matrix. Finally, all of the above signals can be written in a short notation as

$$
\mathbf{y}=\mathbf{h} \mathbf{x}+\mathbf{n} .
$$

We transform equation (7) as the $3 \times 2$ matrix to $6 \times 1$ row vector by insertion complex conjugate operation of equations (2), (4) and (6) yielding

$$
\begin{aligned}
& y_{12}^{*}=h_{11}^{*} x_{5}+h_{12}^{*} x_{6}-h_{13}^{*} x_{4}+h_{14}^{*} x_{3}+n_{12}^{*}, \\
& y_{22}^{*}=h_{21}^{*} x_{5}+h_{22}^{*} x_{6}-h_{23}^{*} x_{4}+h_{24}^{*} x_{3}+n_{22}^{*},
\end{aligned}
$$

and

$$
y_{32}^{*}=h_{31}^{*} x_{5}+h_{32}^{*} x_{6}-h_{33}^{*} x_{4}+h_{34}^{*} x_{3}+n_{32}^{*} \text {. }
$$

Equation (1), (3), (5) and (9)-(11) can be rewritten in a matrix notation as

$$
\left[\begin{array}{l}
y_{11} \\
y_{21} \\
y_{31} \\
y_{12}^{*} \\
y_{22}^{*} \\
y_{32}^{*}
\end{array}\right]=\left[\begin{array}{cccccc}
h_{11} & h_{12} & h_{13} & h_{14} & 0 & 0 \\
h_{21} & h_{22} & h_{23} & h_{24} & 0 & 0 \\
h_{31} & h_{32} & h_{33} & h_{34} & 0 & 0 \\
0 & 0 & h_{14}^{*} & -h_{13}^{*} & h_{11}^{*} & h_{12}^{*} \\
0 & 0 & h_{24}^{*} & -h_{23}^{*} & h_{21}^{*} & h_{22}^{*} \\
0 & 0 & h_{34}^{*} & -h_{33}^{*} & h_{31}^{*} & h_{32}^{*}
\end{array}\right]\left[\begin{array}{c}
x_{1} \\
x_{2} \\
x_{3} \\
x_{4} \\
x_{5} \\
x_{6}
\end{array}\right]+\left[\begin{array}{c}
n_{11} \\
n_{21} \\
n_{31} \\
n_{12}^{*} \\
n_{22}^{*} \\
n_{32}^{*}
\end{array}\right] .
$$

For convenience, we can expressed equation (12) as a compact formulation as

$$
\mathbf{Y}=\mathbf{H X}+\mathbf{N}
$$


where $\mathbf{H}=\left[\begin{array}{cccccc}h_{11} & h_{12} & h_{13} & h_{14} & 0 & 0 \\ h_{21} & h_{22} & h_{23} & h_{24} & 0 & 0 \\ h_{31} & h_{32} & h_{33} & h_{34} & 0 & 0 \\ 0 & 0 & h_{14}^{*} & -h_{13}^{*} & h_{11}^{*} & h_{12}^{*} \\ 0 & 0 & h_{24}^{*} & -h_{23}^{*} & h_{21}^{*} & h_{22}^{*} \\ 0 & 0 & h_{34}^{*} & -h_{33}^{*} & h_{31}^{*} & h_{32}^{*}\end{array}\right]$ is a

channel matrix. $\mathbf{Y}=\left[\begin{array}{llllll}y_{11} & y_{21} & y_{31} & y_{12}^{*} & y_{22}^{*} & y_{32}^{*}\end{array}\right]^{\top}$ is the receive signal vector, $\mathbf{X}=\left[\begin{array}{llllll}x_{1} & x_{2} & x_{3} & x_{4} & x_{5} & x_{6}\end{array}\right]^{\top}$ is the transmit data vector $\mathbf{N}=\left[\begin{array}{llllll}n_{11} & n_{21} & n_{31} & n_{12} & n_{22} & n_{32}\end{array}\right]^{\top}$ is the noise vector.

\section{INTERFERENCE CANCELLATION TECHNIQUES}

In this section, we explain the interference cancellation technique for multi-user detection (MUD) by performing a zero forcing $(\mathrm{ZF})$ receiver, minimum mean square error (MMSE) receiver, parallel interference cancellation (PIC) receiver, and zero-forcing successive interference cancellation (SIC-ZF) receiver, respectively.

\section{A. ZF Receiver}

In order to suppress and reject the interference, we consider the ZF linear receiver. Let $\mathbf{W}_{Z F}$ be the receiver matrix and satisfy the property [12]-[13]

$$
\mathbf{W}_{Z F} \mathbf{H}=\mathbf{I}
$$

where $\mathbf{I}$ is an identity matrix. From (14), $\mathbf{W}_{Z F}=\mathbf{H}^{-1}$ and let the estimated bit is defined as

$$
\hat{\mathbf{X}}=\mathbf{W}_{Z F} \mathbf{Y}
$$

where the received signal is given in (13). After some manipulations, the estimated bit is expressed as

$$
\hat{\mathbf{X}}=\mathbf{X}+\mathbf{H}^{-1} \mathbf{N}
$$

\section{B. MMSE Receiver}

The mean square error (MSE) is defined by [13]-[14]

$$
\begin{array}{ll} 
& \text { MSE }=(\mathbf{X}-\hat{\mathbf{X}})(\mathbf{X}-\hat{\mathbf{X}})^{\top}, \\
\text { but } & \hat{\mathbf{X}}=\mathbf{W}_{\text {MMSE }} \mathbf{Y} .
\end{array}
$$

Substitute (13) into (18) yields,

$$
\hat{\mathbf{X}}=\mathbf{W}_{\text {MMSE }}(\mathbf{H X}+\mathbf{N}) \text {. }
$$

Substitute (19) into (17) yields,

$$
\begin{aligned}
\text { MSE }= & {\left[\mathbf{X}-\mathbf{W}_{\text {MMSE }}(\mathbf{H X}+\mathbf{N})\right]\left[\mathbf{X}-\mathbf{W}_{\text {MMSE }}(\mathbf{H X}+\mathbf{N})\right]^{\top} } \\
= & {\left[\mathbf{X X}^{\top}-\mathbf{X X}^{\top} \mathbf{H}^{\top} \mathbf{W}_{\text {MMSE }}^{\top}-\mathbf{X N}^{\top} \mathbf{W}_{\text {MMSE }}^{\top}-\mathbf{W}_{\text {MMSE }} \mathbf{H X} \mathbf{X}^{\top}+\right.} \\
& \mathbf{W}_{\text {MMSE }} \mathbf{H X X} \mathbf{X}^{\top} \mathbf{W}_{\text {MMSE }}^{\top}+\mathbf{W}_{\text {MMSE }} \mathbf{H X N}^{\top} \mathbf{W}_{\text {MMSE }}-\mathbf{W}_{\text {MMSE }} \mathbf{N X}^{\top} \\
& \left.+\mathbf{W}_{\text {MMSE }} \mathbf{N X}^{\top} \mathbf{H}^{\top} \mathbf{W}_{\text {MMSE }}^{\top}+\mathbf{W}_{\text {MMSE }} \mathbf{N N}^{\top} \mathbf{W}_{\text {MMSE }}^{\top}\right]
\end{aligned}
$$

Since $E[\mathbf{n}]=E\left[\mathbf{n}^{\top}\right]=0, E\left[\mathbf{n n}^{\top}\right]=\sigma^{2}$ and $E\left[\mathbf{x x}^{\top}\right]=E\left[\mathbf{x}^{2}\right]=1$. The ensemble average of (20) is

$$
\begin{aligned}
\mathrm{E}[\mathrm{MSE}]= & \mathbf{1}-\mathbf{H}^{\top} \mathbf{W}_{\text {MMSE }}^{\top}-\mathbf{W}_{\text {MMSE }} \mathbf{H}+\mathbf{W}_{\text {MMSE }} \mathbf{H} \mathbf{H}^{\top} \mathbf{W}_{\text {MMSE }}^{\top} \\
& +\sigma^{2} \mathbf{W}_{\text {MMSE }} \mathbf{W}_{\text {MMSE }}^{\top} .
\end{aligned}
$$

In order to minimize the MSE, we take a derivative of (21) with respect to $\mathbf{W}_{\text {MMSE }}$ and equating to zero. Therefore, the derivative is

$$
\begin{aligned}
\frac{\partial \mathrm{E}[\mathrm{MSE}]}{\partial \mathbf{W}_{\text {MMSE }}}= & \mathbf{0}-\mathbf{H}^{\top}-\mathbf{H}^{\top}+\mathbf{W}_{\text {MMSE }}\left(\mathbf{H H}^{\top}\right)^{\top} \\
& +\mathbf{W}_{\text {MMSE }} \mathbf{H H}^{\top}+\mathbf{W}_{\text {MMSE }} \sigma^{2} \mathbf{I}+\mathbf{W}_{\text {MMSE }} \sigma^{2} \mathbf{I}
\end{aligned}
$$

After some mathematics manipulations, the MMSE receiver is given by

$$
\mathbf{W}_{\text {MMSE }}=\mathbf{H}^{\top}\left[\mathbf{H} \mathbf{H}^{\top}+\sigma^{2} \mathbf{I}\right]^{-1} \text {. }
$$

Substitute (22) into (19), therefore the estimation data bit is

$$
\hat{\mathbf{x}}=\mathbf{H}^{\top}\left(\mathbf{H H}^{\top}+\sigma^{2} \mathbf{I}\right)^{-1}[\mathbf{H} \mathbf{x}+\mathbf{n}] .
$$

Let $\mathbf{A}=\left(\mathbf{H H}^{\top}+\sigma^{2} \mathbf{I}\right)^{-1}$ then

$$
\hat{\mathbf{X}}=\mathbf{X}+\mathbf{H}^{-1} \mathbf{N}
$$

\section{PIC Receiver}

This receiver estimates all of the data bits and then subtracts all signals except the reference user. This process is done and cancelled all the user interference before sending bits to the decision process. If we apply the perfect power control, the best performance is received. In the first step, we transform the original receiver to the decorretaor. The second step, the input bit is calculated from

$$
\hat{\mathbf{X}}=\mathbf{H}^{+} \mathbf{Y}
$$

where $\mathbf{H}^{+}$is the channel matrix and $\mathbf{Y}$ is the received vector, respectively. Next the decision making is done by using

$$
\hat{\mathbf{X}}=\mathbf{Q}(\mathbf{G Y}),
$$


where $\mathbf{Q}$ and $\mathbf{Y}$ denote the slicing function and weighting function, respectively. Let $i$ be the number of transmit antenna, $i=1: 4$, the cancellation process is done by subtracting all interference signals form the desire signal and given by

$$
\mathbf{Y}=\mathbf{Y}-\sum_{j=1}^{3} \mathbf{H}(i, j) \hat{\mathbf{X}}_{j}
$$

The weighting function is calculated by

$$
\mathbf{G}=(\mathbf{H}(:, j))^{+} .
$$

Finally, the estimated data bit has been computed from (28) and (29), then

$$
\hat{\mathbf{X}}_{j}=\mathbf{Q}(\mathbf{G Y})
$$

\section{SIC-ZF Receiver}

Successive interference cancellation zero-forcing (SIC-ZF) employs zero-forcing nulling with ordering cancellation detection. Zero-forcing nulling with ordering cancellation detection performs better than nulling with cancellation, regardless of ordering. This is because the system performance is affected by the detection order of $\mathbf{X}$ components in terms of postdetection SNR when symbol cancellation is used. This is different from nulling alone detection, as each nulling vector $\left(\mathbf{W}_{k}\right)$ is required to be orthogonal to $\mathbf{M}-\mathbf{1}$ rows of $\mathbf{H}$ from (13), according to [15]. However, when symbol cancellation is employed, the nulling vector in $\boldsymbol{i}$-th iteration

$\left(\mathbf{W}_{k i}\right)$ is required to be orthogonal only to the $M-i$ undetected components [16]. A simple consequence of the CauchySchwartz inequality is that the more rows of $\mathbf{H}$ that a particular $\mathbf{W}_{k i}$ is constrained to be orthogonal to, the larger its norm, and thus, according to [17], the smaller its postdetection SNR. The post-detection SNR of modified received vector must be maximized so as to minimize the error propagation as high SNR indicates low symbol error rate. The full SIC ZF detection algorithm [12] can be described compactly as a recursive procedure, including determination of the optimal ordering, as follows:

\section{Initialization:}

Let $i=1$ and $\mathbf{G}_{1}=\mathbf{H}^{+}$, where $\mathbf{G}_{1}$ denotes the MoorePemrose pseudo-inverse of Rayleigh fading channel, $\mathbf{H}^{+}$. Then the values of

$$
\mathbf{K}_{1}=\arg \min _{j}\left\|\left(\mathbf{G}_{1}\right)_{j}\right\|^{2}
$$

specifies the ordering decision in the $i$-th iteration. $\left|\mathbf{G}_{i}\right|_{j}$ presents the $j$-th row of $\mathbf{G}_{i}$ determines the elements of the optimal ordering by selecting the smallest norm of $j$-th row of $\mathbf{G}_{i}$.

\section{Recursion}

Computing respective $\mathrm{ZF}$-nulling vector uses

$$
\mathbf{W}_{\mathbf{k}_{i}}=\left(\mathbf{G}_{i}\right) \mathbf{K}_{i}
$$

Then the decision statistic is written as

$$
\mathbf{r}_{\mathbf{K}_{i}}=\mathbf{W}_{\mathbf{K}_{i}}^{\top} \mathbf{y}_{i},
$$

and the estimated component of $\mathbf{X}$ calculated by

$$
\hat{\mathbf{X}}_{\mathbf{K}_{i}}=\mathbf{Q}\left(\mathbf{r}_{\mathbf{K}_{i}}\right)
$$

Note that $\mathbf{Q}$ is a slicing operation. We perform cancellation of the detected component from the received vector using the relation

$$
\mathbf{y}_{i+1}=\mathbf{y}_{i+1}-\hat{\mathbf{X}}_{\mathbf{K}_{i}}(\mathbf{H})_{\mathbf{K}_{i}} .
$$

Let $\mathbf{G}_{i+1}=\mathbf{H}_{-\mathbf{K}_{i}}^{+}$and computes the new pseudo-inverse for the next iteration. This new pseudo-inverse is based on a deflated version of $\mathbf{H}$, in which columns $\mathbf{K}_{1}, \ldots, \mathbf{K}_{i}$ have been zero. Then

$$
\mathbf{K}_{i+1}=\arg \min _{j}\left\|\left(\mathbf{G}_{1}\right)_{j}\right\|^{2}
$$

determines the elements of the optimal ordering for the next iteration.

\section{Simulation Results}

We perform computer simulations using four transmit antennas and three receive antennas. The modulation scheme is assumed to be binary phase shift keying (BPSK) over Rayleight frequency flat fading channel with perfect channel state information (CSI). We compare the joint optimal detection technique (ZIC-ZF) with ZF, MMSE and PIC receivers. The simulation results are presented in Figure 2. 


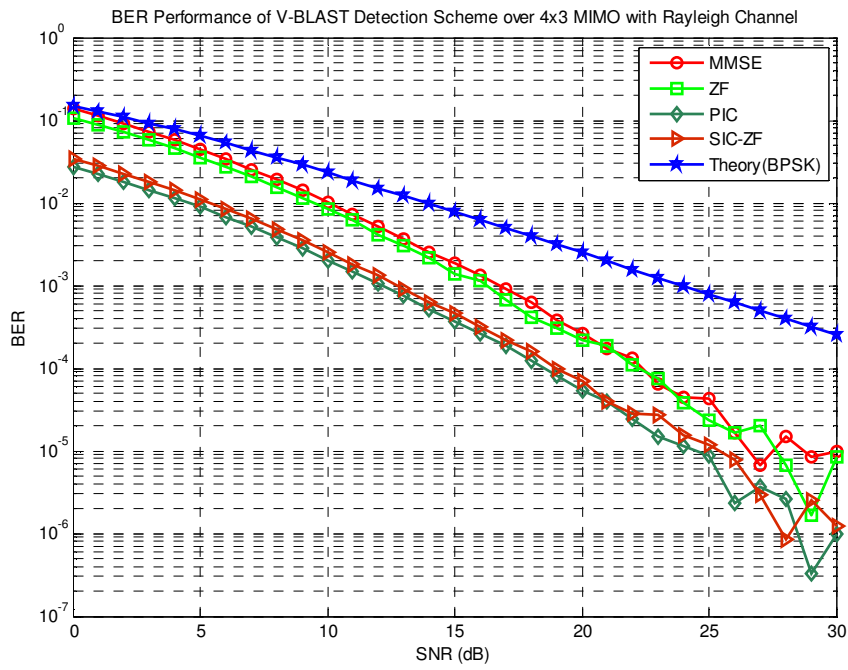

Figure2. Bit Error Rate of V-BLAST/STBC with detection techniques.

We notice that employing the MIMO antennas, VBLAST/STBC, is better than the SISO (classic antenna). We observe that at BER $10^{-3}$, the performance of ZF and MMSE receiver is nearly the same. However, for high value of SNR the MMSE receiver is slightly better. We can observe that the BER performance of the non-linear receivers, SIC-ZF and PIC, outperforms linear receivers (ZF and MMSE). In Figure 2, we notice that PIC has the lowest BER and best performance. Meanwhile, the complexity of this algorithm is significantly reduced as compare with SIC-ZF.

\section{CONCLUSIONS}

In this paper, we described detection techniques of $\mathrm{ZF}$, MMSE, PIC and SIC-ZF over a V-BLAST/STBC over Rayleigh flat fading channel. We notice that the bit error rate performance of MIMO is better the classic SISO. We also observe that the non-linear detector outperforms the linear detector. At BER 10-3 the SNR of SIC-ZF and PIC are 13 and 12 $\mathrm{dB}$, respectively. So PIC yields the best BER performance. It also greatly reduces the complexity and degrades the performance marginally if compare with the algorithm SIC-ZF. This two non-linear detection algorithms can be apply for VBLAST/STBC and MIMO systems.

\section{REFERENCES}

[1] Andrea Goldsmith, Wireless Communications, $1^{\text {st }}$ ed. California: Stanford University, 2004.

[2] M. K. Simon and M. S. Alouni, Digital communication over fading channels, $2^{\text {nd }}$ ed. Hoboken, N.J.: John Wiley\&Sons. 2005.

[3] Biglieri, Ezio et al., MIMO Wireless Communications, $1^{\text {st }}$ ed., United Kingdom: Cambridge University Press., 2007.

[4] P. W. Wolniansky, G. J. Foschini, G. D. Golden and R. A. Valenzuela, "V-BLAST: an architecture for realizing very high data rates over the rich-scattering wireless channel", in Proc. ISSSE, pp. 295-300, 1998.

[5] G. J. Foschini and M. J. Gans, "On the limits of wireless communications in a fading environment when using multiple antennas", Wireless Personal Communications, vol. 6, no. 3, pp. 311335, 1998

[6] E. Telatar, "Capacity of multi-antenna Gaussian channels", European Trans. on Telecommun., vol. 10, no. 6, pp. 585-595, Nov./Dec. 1999.

[7] T. L. Marzetta and B. M. Hochwald, "Capacity of a mobile multiple-antenna communication link in Rayleigh flat fading", IEEE Trans. Inform. Theory, vol. 45, no. 1, pp. 139-157, Jan. 1999.

[8] C. Shen, H. Zhuang, L. Dai, S. Zhou, "Detection algorithm improving V-BLAST performance over error propagation", IEE Electronics Letters, Vol.39, No. 13, pp. 1007-1008, Jun. 2003.

[9] G. J. Foschini, G.D. Golden, R.A. Valenzuela and P.W. Wolniansky "Simplified processing for high spectral efficiency wireless Communication employing multi-element arrays", IEEE J. Selected Areas of Communication, vol. 17,no. 11, pp. 1841-1852, Nov. 1999.

[10] S. Loyka, "V-BLAST outage probability: analytical analysis", in Proc. IEEE VTC Fall Conf., vol. 4, pp. 24-28, Sep. 2002.

[11] N. Prasad and M. K. Varanasi, "Analysis of Decision Feedback Detection for MIMO Rayleigh Fading Channels and Optimum Allocation of Transmitter Powers and QAM Constellations", in Proc. 39th Annual Allerton Conf. on Comm. Control, and Comput., Oct., 2001.

[12] T.S. Rappaport, Wireless Communications: Principles and Practice, $2^{\text {nd }}$ ed., Upper Saddle River, NJ: Prentice Hall, 2002.

[13] J. G. Proakis, Digital Communications, $4^{\text {th }}$ ed., New York: McGrawHill, 2001.

[14] John R. Barry, Edward A. Lee, and David G. Messerschmitt, Digital Communication, ${ }^{\text {rd }}$ ed., United States., 2004.

[15] G.G. Raleigh, and J. M. Cioffi, "Spatio-Temporal Coding for Wireless Communications", in Proc. 1996. IEEE Globecom, pp. 1809-1814, Nov. 1996.

[16] C.Y. Yoon, R. Kohno, H. Imai, "A Spread-Spectrum Multiaccess System with Cochannel Interference Cancellation for Multipath Fading Channels", IEEE J. Selected Areas of Communications, vol. 11, pp. 1067-1075, Sep., 1993.

[17] A.L.C. Hui, K.B. Letaief, "Successive Interfence Cancellation for Multiuser Asynchronous DS/CDMA Detectors in Multipath Fading Links", IEEE Trans. Comm. vol. 46, no. 3, March, 1998. 